\title{
Identificación del mezclador de gases de la nueva incubadora neonatal BAN
}

\section{RESUMEN}

El objetivo del trabajo presentado fue el desarrollo de un método de Identificación de Sistemas aplicado al mezclador de gases de la incubadora neonatal BAN, con la finalidad de encontrar un modelo matemático que describa su dinámica para implementar posteriormente un controlador. El método empleado consideró la realización de una Identificación $\mathrm{No} \mathrm{Pa}$ ramétrica, la cual consistió en excitar la entrada del mezclador con señales generadas por una computadora para poder determinar información básica pero valiosa. Luego, se realizó la Identificación Paramétrica que consistió en excitar la entrada del mezclador con una señal binaria seudo aleatoria para obtener la matriz de datos, con ellos se obtuvieron los coeficientes del modelo ARX. Las pruebas de validación cruzada y correlación efectuada s mostraron la efectividad del método. Basado en los resultados del estudio del mezclador, se concluye que el subsistema Ducto de Oxígeno es de cuarto orden considerando los actuadores acoplados mecánicamente y el subsistema Ducto de Aire es de cuarto orden considerando el actuador y el transmisor.

Palabras clave: Modelo ARX, Identificación de Sistemas, Método de mínimos cuadrados, validación cruzada.

GAS MIXER IDENTIFIER OF THE NEW BAN NEONATAL INCUBATOR

\section{ABSTRACT}

The objective of this work is to develop a method of Systems Identification applied to the gas mixer in the neonatal incubator BAN, with the aim of finding a mathematical model that describes its dynamics to implement later a controller. The method considered the conduct of an identification nonparametric which was to excite the entrance to the mixer with signals generated by a computer to determine basic information but valuable. Then, the parametric identification was made consisting of excite the entrance to the mixer with a pseudo-random binary signal to obtain the data matrix with which the coefficients were obtained model ARX. The validation and cross correlation conducted showed the effectiveness of the method. Based on the survey results mixer, it is concluded that the subsystem Pipeline Oxygen is the fourth order considering actuators mechanically coupled and transmitter and subsystem Pipeline Air is the fourth order considering the actuator and transmitter.

KEYwords: Model ARX, System Identification, Least squares method, Cross validation.

\section{INTRODUCCIÓN}

La necesidad de un mejor equipamiento que pueda apoyar satisfactoriamente a un sector poco atendido de la población como son los neonatos prematuros justifica el desarrollo de alternativas tecnológicas como el mezclador de gases para las incubadoras para suministrar un porcentaje adecuado de oxígeno y resolver problemas de extrema gravedad como el nacimiento prematuro. Esta deficiencia coadyuva a la alta tasa de mortalidad infantil de cerca de 35 de cada 1000 nacidos (Díaz L. et al., 2003), en la cual los neonatos constituyen un alto porcentaje. La carencia de incubadoras impide ampliar la cobertura de atención y las deficiencias técnicas en el diseño de las incubadoras convencionales dejan algunas veces secuelas irreversibles en los recién nacidos, tal como la fibroplasia retrolental o ceguera causada por la administración inadecuada de oxígeno (AENOR, 1997). El control de la cantidad de oxígeno administrado es importante porque demasiado oxígeno puede ocasionar daño a la retina. A pesar de las consecuencias de no administrar cuidadosamente la dosis de oxígeno a los niños prematuros, este se suministra inadecuadamente debido a la falta de mezcladores de aire-oxígeno adecuados (Sola A. et al., 2005). El alto uso de oxígeno, el filtrado excesivo del flujo de aire y el tiempo muerto para la desinfección que usualmente es de una semana a un mes ocasiona la elevación del costo operativo de la incubadora.

El habitáculo que aloja al neonato no es completamente estéril, por lo que aumenta la posibilidad de contagio de alguna enfermedad por contacto con un ambiente contaminado. Por otro lado, las incubadoras no pueden brindar tratamientos de oxigenación por sí mismas, tienen por ello que emplearse equipos adicionales, elevando los costos de atención y finalmente, el ruido en el habitáculo puede ser muy elevado pudiéndole provocar estrés y problemas en su desarrollo. La baja cobertura de atención neonatal en el Perú se puede paliar con una mayor inversión por parte del Estado; pero las deficiencias técnicas del diseño convencional sólo podrán ser subsanadas por mejores conceptos o principios de funcionamiento.

La incubadora CPAP es un equipo para el soporte respiratorio en pacientes neonatales y pediátricos, usado para la administración de presión positiva nasal continua de las vías respiratorias en

1 Grupo de Investigación GIDEMS. Sección Electricidad y Electrónica. Facultad de Ciencias e Ingeniería, Departamento de Ingeniería. Pontificia Universidad Católica del Perú, Av. Universitaria 1801, Lima 32, Perú. 
recién nacidos prematuros con insuficiencia respiratoria SDR. El sistema opera con flujo continuo de presión limitada en modo CPAP permitiendo la máxima estabilidad y el control de la presión, flujo de aire, oxígeno, humedad y temperatura, a través de una cánula NCPAP convencional. La manipulación del flujo de aire y del flujo de oxígeno mediante un Mezclador para obtener la concentración de la mezcla resultante es importante y antes de construir el controlador se debe conocer el modelo del Mezclador.

Como se tiene poco conocimiento del sistema, el método empieza por una implementación experimental para obtener una respuesta transitoria o una respuesta en frecuencia que posibilite conseguir una información básica pero importante de la dinámica y de las perturbaciones. Basándose en los resultados, como constante de tiempo, puntos de inflexión, orden del sistema, intervalo de muestreo apropiado se puede mejorar la estructura del modelo y pueden ser necesarios nuevos experimentos. En la práctica, la identificación de sistemas es un proceso iterativo. Un requisito de la señal de entrada es que debe excitar de forma suficiente todos los modos del proceso y debe por tanto, ser insensible a las características de la señal de entrada.

Las estructuras del modelo se obtienen del conocimiento previo del proceso y de las perturbaciones. En algunos casos, el único conocimiento previo que se tiene es que el proceso se puede describir como un sistema lineal en un rango de operación concreto, siendo natural utilizar representaciones de sistemas lineales de tipo general como los modelos de caja negra.

Cuando se formula un problema de identificación se introduce un criterio para tener una medida de hasta qué punto un modelo se ajusta a los datos experimentales. Con frecuencia los criterios para los sistemas discretos se expresan en la forma cuadrática, pero puede ser de otras formas. El principio de los mínimos cuadrados es un método basado en la minimización de la suma de los cuadrados del error, este método de los mínimos cuadrados está restringido a estructuras de modelo que son lineales en los parámetros desconocidos.

La estimación de parámetros se puede formular como un problema de optimización, en el que el mejor modelo es aquel que mejor se ajusta a los datos de acuerdo con un criterio. Existe una variedad de modelos paramétricos que se ajustan a la estructura general de la forma:

$$
y(t)=\frac{B\left(q^{-1}\right)}{A\left(q^{-1}\right)} q^{-\lambda t} \cdot u(t)+\frac{D\left(q^{-1}\right)}{C\left(q^{-1}\right)} \cdot e(t)
$$

(ecuación 1)
Donde: $u(t), y(t)$ y $u(t)$ son la entrada, salida y ruido del sistema respectivamente, $n k$ el retardo del sistema y $A, B, C, D$ son los polinomios en función del operador desplazamiento $\left(q^{-1}\right)$. Escoger una estructura significa definir los órdenes de todos los polinomios que intervienen, en muchas ocasiones esta elección lleva a simplificaciones típicas de la estructura general anterior, entre las que hay que destacar: ARX, ARMAX, OE, BJ, etc.

El modelo ARX llamado ecuación del error y sus polinomios están definidos por la ecuación 2 y 3 respectivamente:

$$
\begin{aligned}
& A\left(q^{-1}\right) * y(t)=B\left(q^{-1}\right) q^{-n k} * u(t)+e(t) \\
& A(q)=1+a_{1} * q^{-1}+\ldots+a_{n a} * q^{-n} \\
& B(q)=b_{1} * q^{-1}+\ldots+b_{n b} * q^{-n b}
\end{aligned}
$$

Donde: $A\left(q^{-1}\right)$ y $B\left(q^{-1}\right)$ son los polinomios del modelo en función del operador desplazamiento $q^{-1}, a_{1} \ldots$ $a_{n a} b_{1} \ldots b_{n b}$ los coeficientes y la estructura está definida por $n a$ el número de polos, $n b$ el número de ceros más uno.

$\Phi(k)=[-y(k-1) \ldots-y(k-n) u(-1-d) \ldots u(k-n-d)]^{t}$

(ecuación 4)

La identificación paramétrica implica conocer la respuesta a la señal seudo binaria aleatoria (SBPA) que permite obtener la matriz de datos de trabajo. Generalmente, se requiere un tratamiento previo de los datos para hacerlos más adecuados al proceso de identificación, como por ejemplo: aplicar filtrado, eliminar valores medio, eliminar transitorios indeseables, etc. Todo proceso de identificación parte de un conjunto de datos obtenidos de forma experimental del sistema que se pretende modelar usándose, generalmente la mitad de $\mathrm{N}$ para realizar la identificación, y los datos restantes se reservan para realizar la validación del mismo.

El resultado obtenido son dos ecuaciones en diferencias que representan el modelo paramétrico tanto del ducto de aire como del ducto de oxígeno del mezclador de gases, objeto de estudio.

\section{MATERIALES Y MÉTODOS}

La instalación experimental utilizada para la recolección de datos necesarios para la obtención del modelo matemático del mezclador de gases de la incubadora BAN, consta de: un moto-reductor, un restrictor, un ducto de plástico corrugado y un 
transmisor de flujo para el subsistema ducto de oxígeno; un compresor, un ducto de plástico corrugado y un transmisor de flujo para el subsistema ducto de aire; ambos transmisores son Honeywell AWM5104N y ambos susbsistemas utilizan programas de aplicación en MATLAB® y SIMULINK@ desde una PC PII.

La identificación no paramétrica empezó con un experimento de ganancia estática con la finalidad de evaluar la zona de trabajo lineal para ambos subsistemas, en la operación del motorreductor-restrictor se consideró una acción de control a partir de $30 \%$ de apertura y en el compresor una acción de control a partir de 2.50 v. Los resultados obtenidos del experimento de ganancia estática se muestran en la Figura 1.

Luego se realizó un experimento de respuesta a la señal escalón, consistente en variar tanto el voltaje del compresor como el voltaje del motorreductorrestrictor a la entrada del mezclador dentro de la zona de trabajo y observar la variación del flujo de aire y oxígeno. Los resultados obtenidos del experimento de respuesta al escalón se muestran en la Figura 2.

Para la identificación paramétrica se realizó otro experimento que consistió en generar una señal SBPA capaz de excitar todos los modos de ambos subsistemas para obtener la matriz de datos de trabajo entrada-salida [u(k) y(k)]. Los resultados del experimento de respuesta a la señal SBPA se muestran en la Figura 3.
Los valores observados son tratados para inicializar la identificación utilizando los mínimos cuadrados para el cálculo del error. El proceso de identificación del modelo matemático utiliza los primeros $75 \%$ de los datos obtenidos. Con la finalidad de visualizar la dinámica del mezclador objeto de estudio, se presenta un diagrama de ubicación de polos y ceros usando un periodo de muestreo de 0.01s. y 0.05s. Los resultados se muestran en la Figura 4 . Con el $25 \%$ final de los datos (que no fueron usados para la identificación) se realiza la prueba del modelo, es decir el proceso de validación cruzada la cual permite mostrar el grado de ajuste entre el modelo y los datos observados. Los resultados de la validación para ambos subsistemas se muestran en la Figura 5 y 6.

\section{RESULTADOS Y DISCUSIÓN}

Se presenta los resultados de los experimentos conducentes a la obtención del modelo matemático del mezclador de gases de la incubadora neonatal BAN, mediante la identificación no paramétrica y luego la identificación paramétrica. Los resultado del experimento de ganancia estática de ambos subsistemas se muestran en la figura 1.

Los resultados obtenidos del experimento de respuesta al escalón para ambos subsistemas se muestran en la Figura 2. Se puede apreciar que el retardo en el Ducto de Oxígeno es de 0.05 s. mayor que el retardo en el Ducto de Aire el cual es de $0.03 \mathrm{~s}$. debido a que presenta los actuadores acoplados mecánicamente.

FIGURA 1. RESULTADO DEL EXPERIMENTO DE GANANCIA ESTÁTICA DE AMBOS SUBSISTEMAS
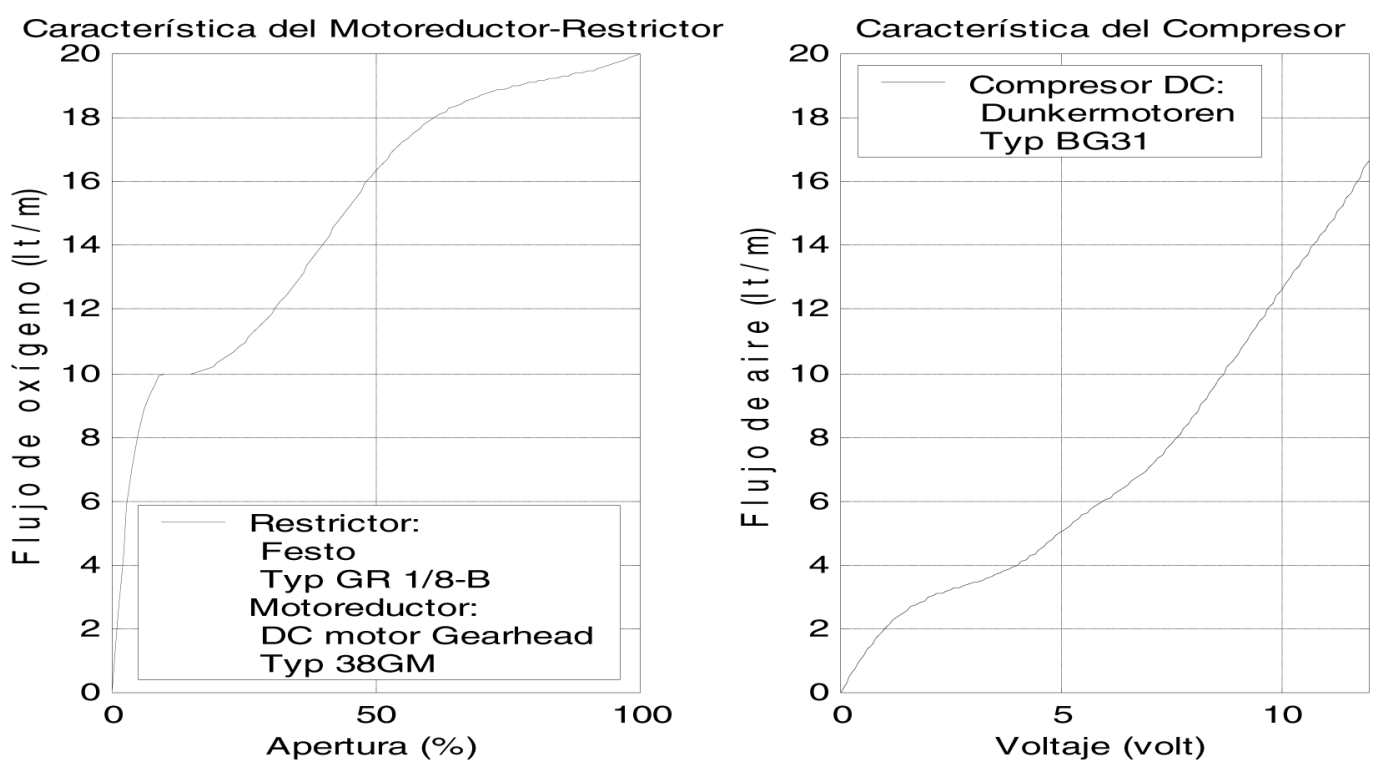

Fuente: Elaboración propia. 


\section{FIGURA 2. RESULTADO DEL EXPERIMENTO RESPUESTA A LA SEÑAL ESCALÓN} PARA AMBOS SUBSISTEMAS
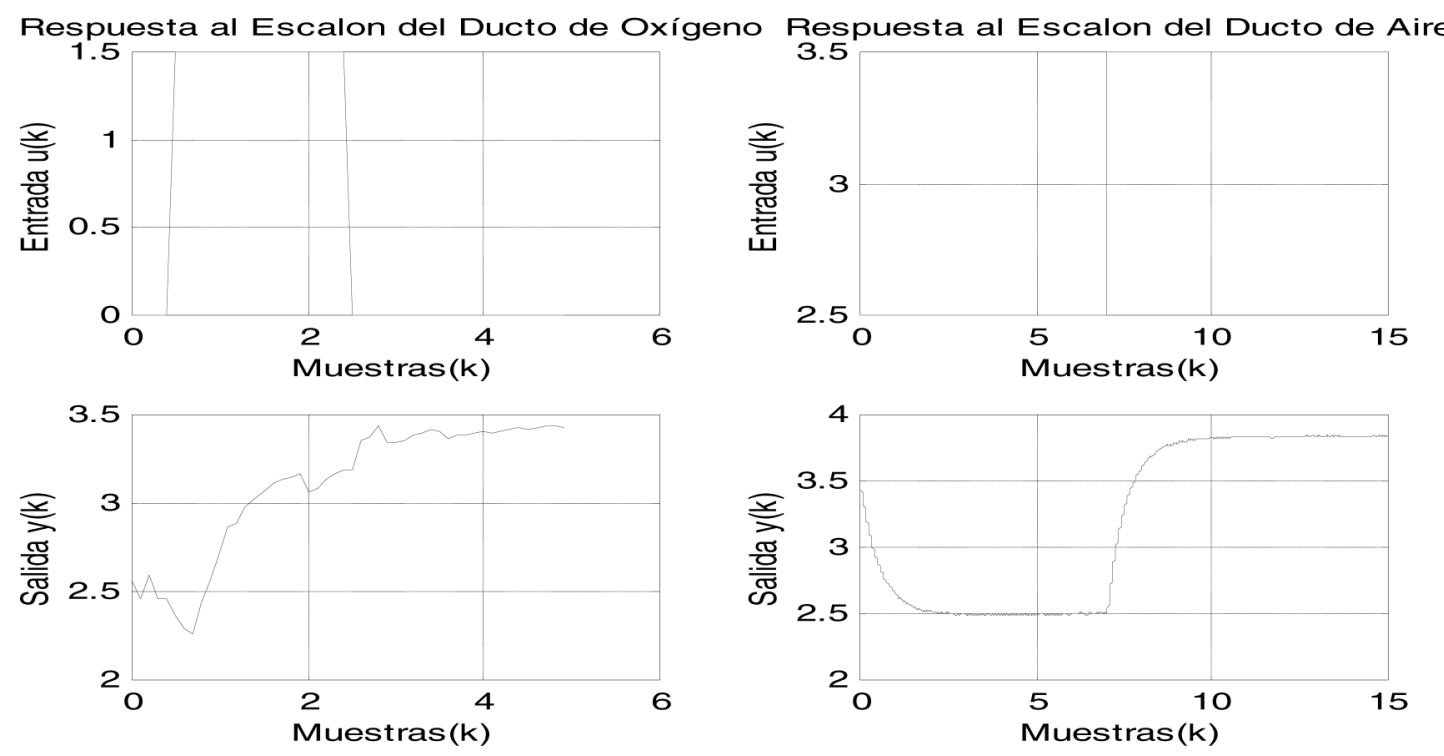

Fuente: Elaboración propia.

Debe tenerse en cuenta que el Ducto de Oxígeno es un subsistema que consta de un motorreductor acoplado a un restrictor que controla el flujo que circula por una tubería y que usa un transmisor para medir el flujo. Asimismo, el Ducto de Aire es otro subsistema que consta de un compresor que suministra el aire que circula por una tubería y que usa otro transmisor para medir el flujo.

Los resultados del experimento de respuesta a la señal SBPA para ambos subsistemas se muestran

en la Figura 3. Los datos posibilitaron el análisis espectral y de correlación para evaluar la bondad de la identificación en retardo, periodo de muestreo, orden, etc.

Los polos y ceros de la función de transferencia en tiempo discreto para los subsistemas ducto de oxígeno y ducto de aire respectivamente se muestran en la Figura 4. Se puede observar que la estructura de modelo para el ducto de oxígeno es [ [ $\left.\begin{array}{lll}4 & 4 & 1\end{array}\right]$ y para el ducto de aire es [4 4 1].

FIGURA 3. RESULTADO DEL EXPERIMENTO DE RESPUESTA A LA SEÑAL SBPA DE AMBOS SUBSISTEMAS
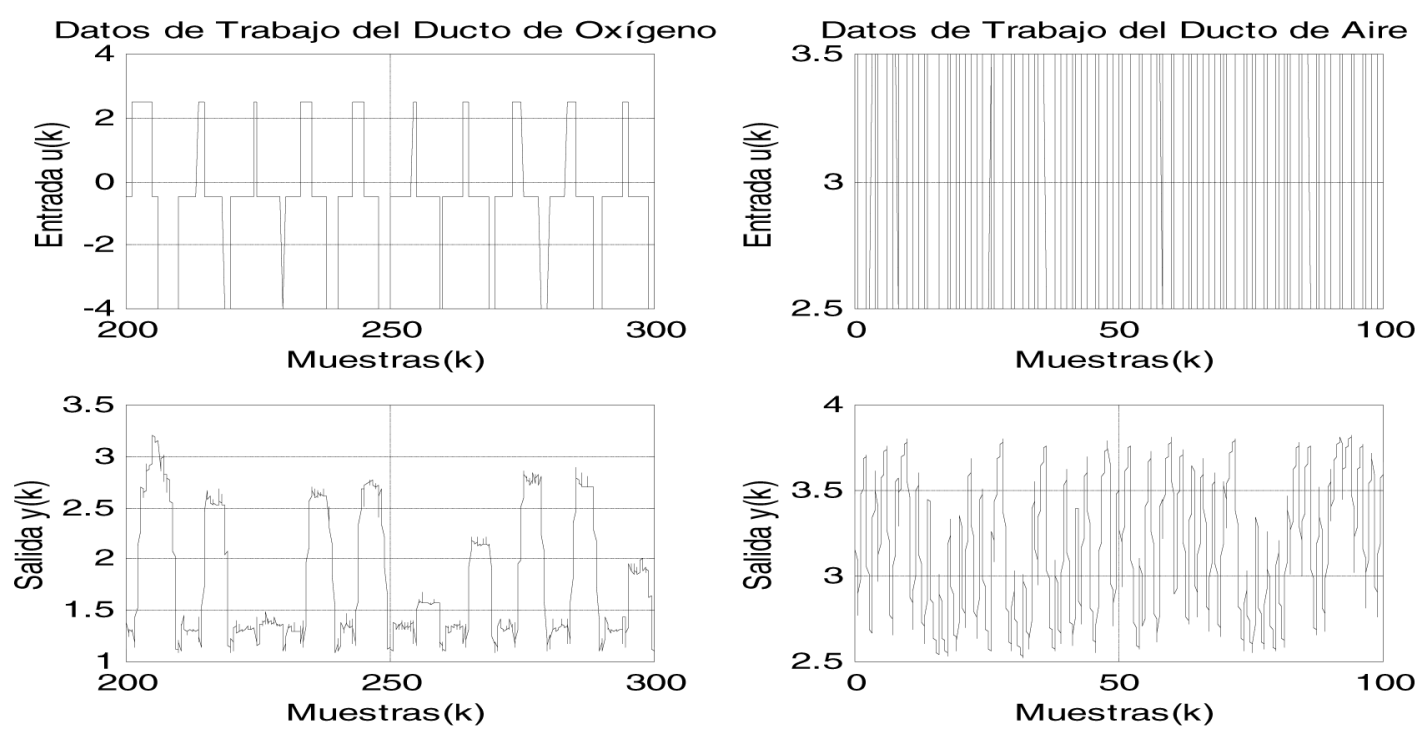
FIGURA 4. DIAGRAMA DE UBICACIÓN DE POLOS Y CEROS PARA AMBOS SUBSISTEMAS.
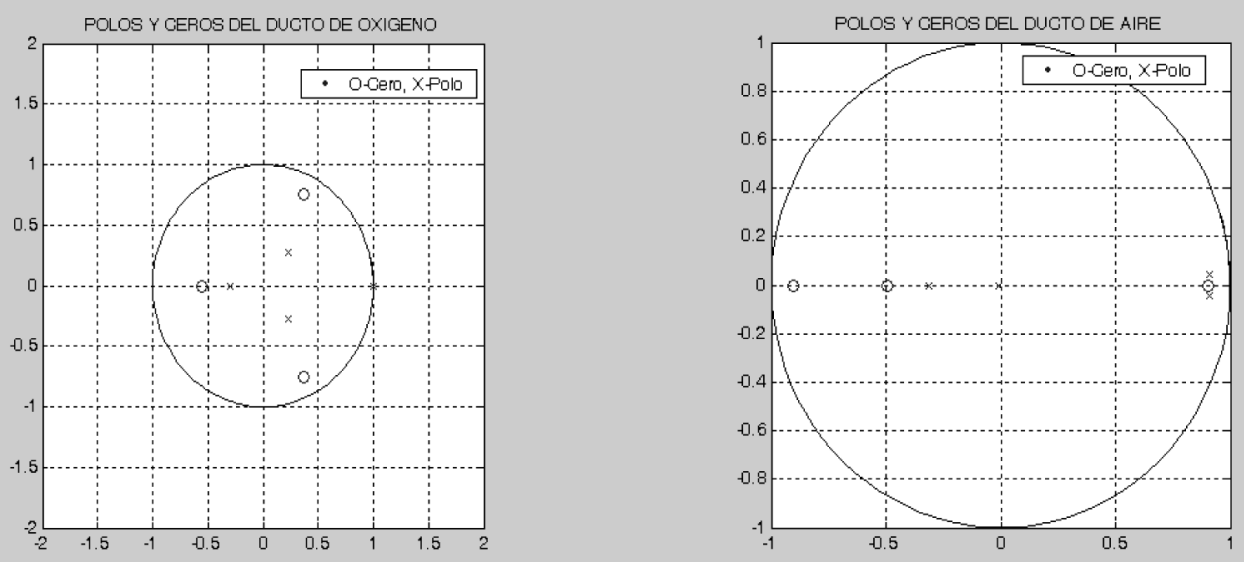

FIGURA 5. RESULTADO DE LA VALIDACIÓN CRUZADA PARA EL MODELO DEL DUCTO DE OXÍGENO
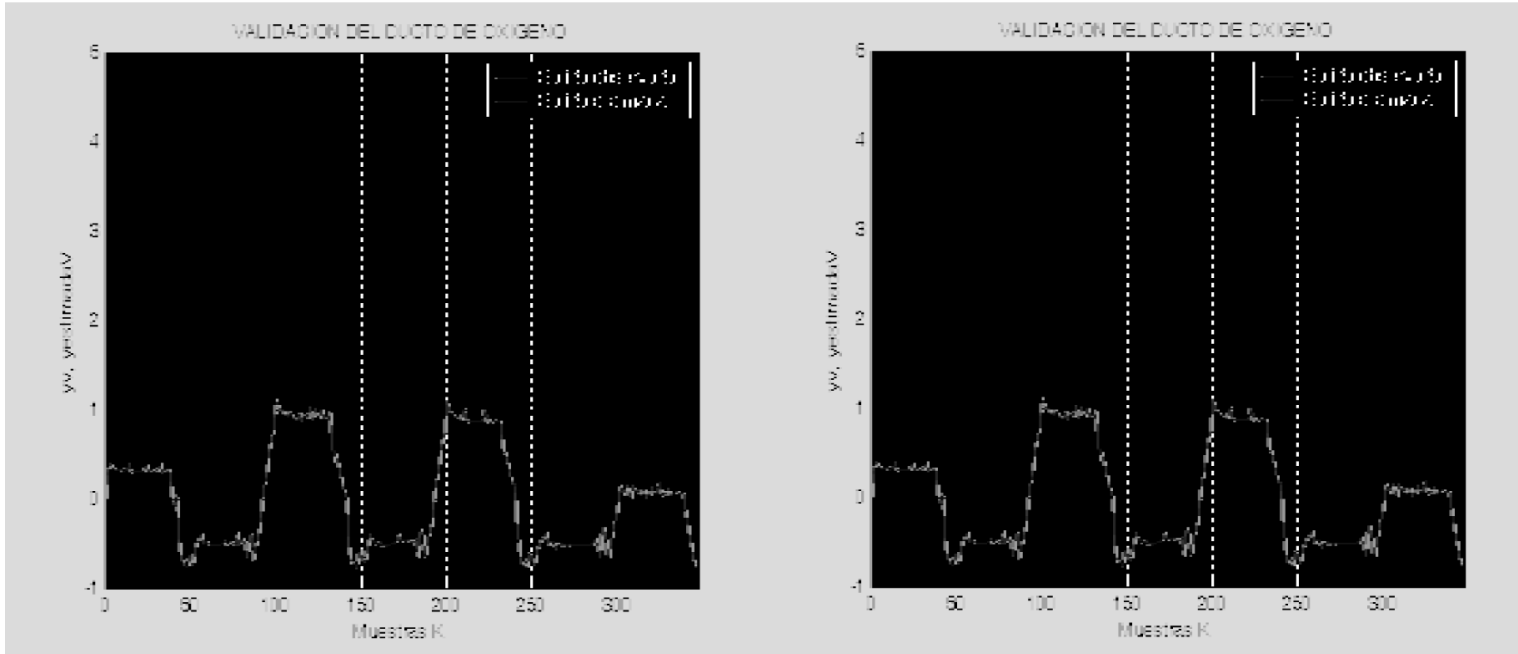

FIGURA 6. RESULTADO DE LA VALIDACIÓN CRUZADA PARA EL MODELO DEL DUCTO DE AIRE.

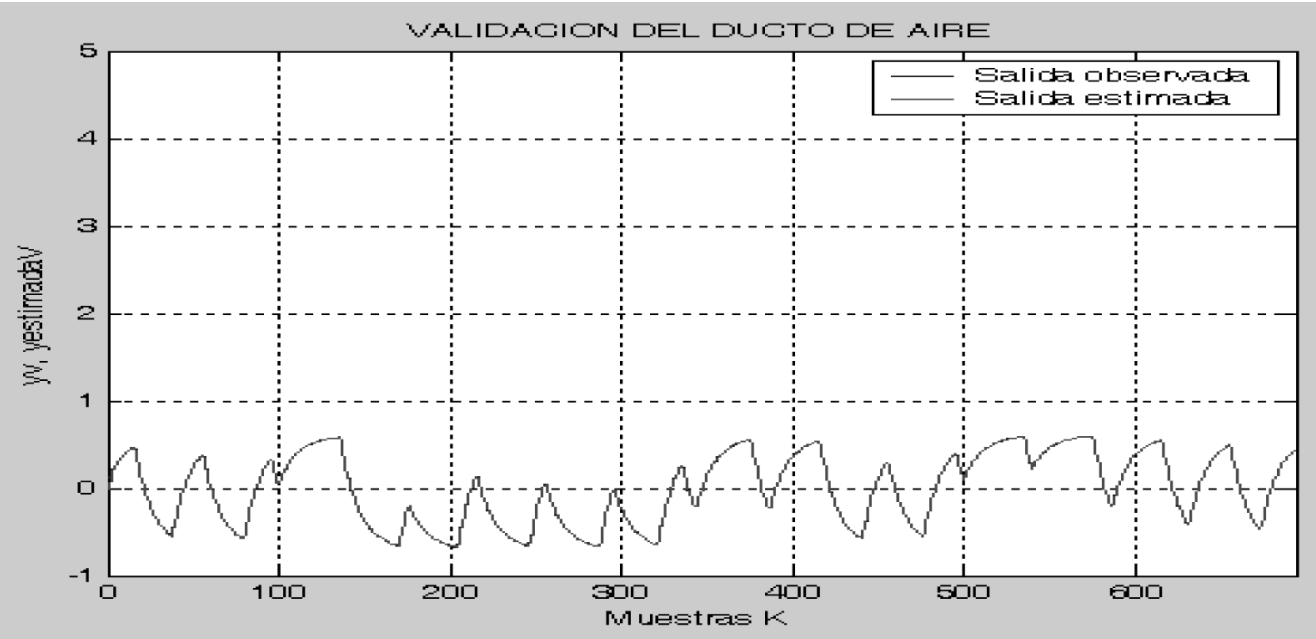


Los resultados de la prueba de validación cruzada tanto del subsistema ducto de oxígeno como del ducto de aire muestran el buen ajuste entre los datos observados y los datos del modelo. Los resultados de la validación por simulación para ambos subsistemas se muestran en las Figuras 5 y 6.

El estudio ha posibilitado obtener el modelo matemático que responde a las siguientes ecuaciones en diferencias. Para el subsistema ducto de oxígeno el modelo es:

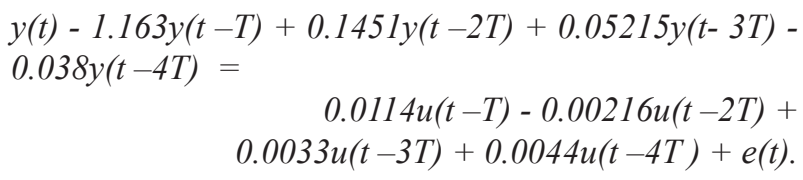

Para el subsistema ducto de aire el modelo es:

$y(t)-1.499 y(t-T)+0.2502 y(t-2 T)+0.2605 y(t-3 T)+$ $0.0017 y(t-4 T)=$

$0.0525 u(t-T)+0.0262 u(t-2 T)-$ $0.0428 u(t-3 T)-0.0213 u(t-4 T)+e(t)$.

Donde:

$y(t-n T)$ es la salida $n$ periodos de muestreo desplazada,

$u(t-n T)$ es la entrada $n$ periodos de muestreo desplazada,

$e(t)$ es el residuo del modelo.

\section{CONCLUSIONES}

Se ha presentado un método para obtener el modelo matemático que explica la dinámica de los subsistemas del mezclador de gases de la incubadora neonatal BAN basado en la identificación de sistemas dinámicos.

El retardo, el periodo de muestreo, la estructura de modelo y los coeficientes que parametrizan el modelo, fueron explicados. El modelo ARX fue empleado por su sencillez y explicación del comportamiento dinámico del mezclador objeto de estudio, en ambos casos la estructura fue [4 4 1]. Los coeficientes se estimaron utilizando el criterio de mínimos cuadrados y el ajuste FIT se obtuvo de las herramientas del Matlabß, los cuales mostraron los residuos más bajos.

La identificación no paramétrica y paramétrica fue completa y con la validación final se pudo apreciar la efectividad del modelo que será usado en el diseño del controlador.

\section{REFERENCIAS}

AENOR UNE-EN-60601-2-21, Asociación Española de Normalización y Certificación, Madrid, España (1997).

Astrom K.J. and Wittenmark. B. (1997). ComputerControlled Systems. Theory and Design, Third Edition, Prentice Hall.

Díaz Ledesma, L., Huamán Guerrero, M., Necochea Villafuerte, I. et al. Mortalidad perinatal en el Hospital Nacional Edgardo Rebagliati Martins. Revista Médica Herediana [online]. Set. 2003, vol. 14, no.3 [citado el 05 enero 2007], pp. 117-121. Disponible en: http://www.scielo. org. pe/scielo. php? script=sciarttext pid=S1018$130 \times 2003000300004 \&$ Ing=es\&nrm=iso. ISSN 1018-130X.

Goodwin G. C., Gevers M., and Ninnes B. (1992). Quantifying the Error in Estimated Transfer Functions with Application to Model Order Selection, IEEE Transaction Automatic Control, Vol. 37, No. 7, pp. 913-929.

Ljung L. (1996). Development of System Identification, 13 th Triennial World Congress, San Francisco, USA., pp. 141-146.

Mendel J. M. (1973). Discrete Techniques of Parameter Estimation. The Equation Error Formulation, Marcel Dekker, N.Y.

Peterka V. (1981). Bayesian Approach to System Identification, In: Trends and Progress in System Identification, Pergamon Press, Elmsford, N. Y.

Poolla K., Khargonekar. P., Krause J. and Nagpal K. (1994). A Time-Domain Approach to Model Validation, IEEE Transaction Automatic Control, AC-39, pp.951-959.

Reiersol O. (1941). Confluence Analysis by Means of Lag Moments and Other Methods of Confluence Analysis, Econometrica, No 9, pp. 1-23.

Soderstrom T. and Stoica P. (1989). System Identification, Prentice Hall.

Sola A., Chow L., Rogido M. (2005) Retinopatía de la prematuridad y oxigenoterapia: una relación cambiante, Anales de Pediatría (Asociación Española de Pediatría), 62(1), pp 48-63. 